\title{
Antioxidant Effects of Seminal Plasma on Cellular Morphological Viability of Swine Semen Post-Cryopreservation
}

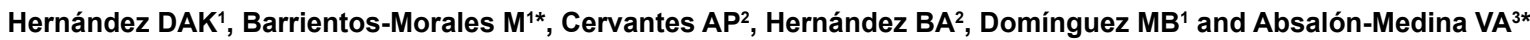

${ }^{1}$ Laboratorio de Biología de la Reproducción Animal, Facultad de Medicina Veterinaria y Zootecnia, Universidad Veracruzana, Veracruz Llave, Veracruz 91710 , México ${ }^{2}$ Laboratorio de Biología Celular. Facultad de Medicina Veterinaria y Zootecnia, Universidad Veracruzana, Veracruz Llave, Veracruz 91710, México

${ }^{3}$ Department of Clinical Studies Section of Reproduction and Behavior, School of Veterinary Medicine, University of Pennsylvania New Bolton Center, Kennett Square 19348, USA

\begin{abstract}
The aims of this study were to measure the antioxidant enzyme activity of superoxide dismutase (SOD) and glutathione peroxidase (GPX1) in seminal plasma (SP), the influence of temperature overtime on such enzymes and, to assess post-thaw viability of semen supplemented with autologous or homologous SP by eosin-nigrosin (EN) and hypoosmotic tests (HOST). A total of 48 sperm-rich fractions from 8 boars were collected and equally divided for SP antioxidant activity determination or cryopreservation experiments. Marked differences in SP antioxidant activity were found amongst individuals. SOD values ranged from $7.88 \pm 0.04 \mathrm{U} / \mathrm{g}$ to $12.01 \pm 0.07 \mathrm{U} / \mathrm{g}$. Restricted maximum likelihood variance components estimates (REML) indicated that $98 \%$ of the variation resided between individuals $(\mathrm{p}<0.05)$. In addition, GPX1 activity ranged from $0.03 \pm 0.001 \mathrm{U} / \mathrm{g}$ to $0.05 \pm 0.005 \mathrm{U} / \mathrm{g}$ with a REML between and within individual of $58 \%$ and $42 \%$, respectively $(p<0.05)$. Further, temperature largely influenced SOD and GPX1 activity (Spearman's $\rho \geq 0.77$; main effect $p<0.01$ ). Regardless, sperm viability improved significantly in groups supplemented with $20 \%$ (v/v) SP compared with control as per EN $27 \pm 0.59 \%$ vs. $26 \pm 0.23 \%$ or HOST $28 \pm 0.27 \%$ vs. $18 \pm 0.27 \%(p<0.05)$. Although there was an additive effect of SP on sperm viability, the antioxidant levels were not strongly correlated to sperm morphology. Therefore, other factors in seminal plasma are contributing to sperm viability and overall fitness towards a successful fertilization.
\end{abstract}

Keywords: Seminal plasma; Antioxidant; Sperm viability; Cryopreservation

\section{Introduction}

Successful swine fertility programs can be achieved using extended cooled semen when compared to those using natural service. Although the former is a routine procedure, its use is limited because sperm cells remain viable for about 3 to 5 days [1]. Cryopreservation would offer a solution to the erstwhile problem by long-term storage. However, post-thaw viability of cryopreserved semen in swine species is generally compromised with an inherent reduction in reproductive performance [2]. Reportedly, boar sperm has been found particularly sensitive to cryoinjuries compared with other species. During freezing about $50 \%$ of sperm cells lose viability due to several factors such as heat shock, intracellular ice crystal formation, oxidative stress and cryocapacitation $[3,4]$ that negatively affects fertility rates $[5,6]$; effects include motility impairment, chromatin damage and membrane alterations like decreased mitochondrial membrane potential [6,7]. Consequently, these cellular changes may potentially shorten the life span of spermatozoa by modifying regulatory pathways such as those of the acrosome reaction and thus, leaving a significant portion of the sperm population unable of interacting with the oviduct or ultimately of fertilizing the eggs [8].

A body of evidence indicates that seminal plasma (SP) ameliorates cryoinjuries and likely extends sperm longevity by delaying the undesired early onset of capacitation and acrosome responsiveness [5,6,9-11]. The protective effects of SP may be attributed to critical components such as antioxidant enzymes and other stabilizing proteins capable of modulating sperm function [12-14]. However, SP is removed prior cryopreservation to dilute the semen at a specific concentration in the extender. This dilution effect on SP may result in oxidative damage to unprotected membranes hence, compromising sperm integrity [15]. Additionally, dead spermatozoa contribute towards the cumulative
ROS levels, exacerbating the process of damage to viable sperm [1618]. Furthermore, controversial results may hamper the practical use of SP on cryopreserved semen i.e., while some studies support beneficial effects of SP [19-22], others are confounded by intricate experimental designs [23-25] or even detrimental effects have been reported [26,27]. It is plausible to infer that the variation in SP antioxidant activity between boars may carry a significant weight since adding SP from good freezers to frozen-thawed semen with mediocre performance resulted in improved viability and membrane integrity [23].

One of the attributes of SP is the antioxidant activity from critical enzymes such as glutathione peroxidases (particularly GPX1), superoxide dismutases (SOD) and catalases (CAT). Glutathione peroxidases are key players in one of the mayor eukaryotic redox systems i.e., glutathione, by keeping a balance on its reduced (GSH) and oxidized (GSSG) forms [28]. Additionally, SOD catalyzes the transfer of electrons to free radicals resulting in $\mathrm{O}_{2}$ or $\mathrm{H}_{2} \mathrm{O}_{2}$ and CAT degrades

*Corresponding authors: Absalón-Medina VA, Department of Clinical Studies Section of Reproduction and Behavior, School of Veterinary Medicine, University of Pennsylvania New Bolton Center, Kennett Square 19348, USA, Tel: 610-9256571; E-mail: vabsa@vet.upenn.edu

Barrientos-Morales M, Laboratorio de Biología de la Reproducción Animal, Facultad de Medicina Veterinaria y Zootecnia, Universidad Veracruzana, Veracruz Llave, Veracruz 91710, México

Received February 13, 2015; Accepted March 24, 2015; Published March 27, 2015

Citation: Hernández DAK, Barrientos-Morales M, Cervantes AP, Hernández BA Domínguez MB, et al. (2015) Antioxidant Effects of Seminal Plasma on Cellular Morphological Viability of Swine Semen Post-Cryopreservation. J Veterinar Sci Technol 6: 225. doi:10.4172/2157-7579.1000225

Copyright: (c) 2015 Hernández DAK, et al. This is an open-access article distributed under the terms of the Creative Commons Attribution License, which permits unrestricted use, distribution, and reproduction in any medium, provided the original author and source are credited. 
hydrogen peroxide excess. Altogether, these enzymes are considered the main barrier as per sperm antioxidant protection [14] and their concentrations may not only have an effect at cryopreservation but on the overall boar fertilizing ability [3,29]. Despite some discouraging results of SP as an additive for cryopreservation, literature supporting its beneficial effects is outweighing e.g., protection against cold shock, reduction of cryocapacitation $[5,6,14,15]$ and, improvement on the percentage of viability, motility and integrity of both plasma and acrosome membrane $[23,30,31]$. Recently, a seasonal variation of antioxidant activity in fluids from the vesicular glands has been reported and intriguingly, this effect is independent from the antioxidant enzyme activity of epididymal fluids [32]. The aim of this study was 1) to measure the levels of enzymatic activity of SP antioxidants during the hot weather in Tropical Mexico and 2) to evaluate the effect of the addition of either autologous or homologous SP to frozen-thawed semen on morphological viability.

\section{Material and Methods}

\section{Reagents and media}

Unless otherwise stated, chemicals used in this study were purchased from Sigma-Aldrich Co. (St. Louis, MO, USA).

\section{Experimental design}

Procedures involving animals were approved by the Institutional Animal Care and Use Committee at the University of Veracruz, Mexico. Boar selection was based on the following criteria: two to four years old, clinically healthy, without pathologies associated to the male reproductive tract and with a proven history of fertility after conventional AI with fresh extended semen. In addition, boars followed a routine schedule of vaccination, deworming, and vitamins (mainly $\mathrm{ADE}$ ) appropriate to the species. Boars were housed in individual pens, fed twice daily with $1.5 \mathrm{~kg}$ of concentrate that contained $15 \%$ of crude protein and 1.2 Mcal of metabolizable energy. The overall project is divided in two main sections: 1) Extraction of seminal plasma with corresponding antioxidant enzyme activity measurements and, 2) Sperm morphological viability determination post-thaw. The experimental design is summarized in Figure 1. Additionally, average temperature at the time of ejaculate collection was recorded in order to measure its effects on SP antioxidant activity (Figure 2).
Experiment 1: Antioxidant enzyme activity of seminal plasma: Frozen samples of SP corresponding to each ejaculate from each boar were allocated to measure the enzyme activity of GPX1 and SOD, expressed in units per gram $(\mathrm{U} / \mathrm{g})$ of total protein using a randomized analysis of variance where boar was the random variable to account for between-and-within variation of SP of each individual. The goal of this experiment was to pinpoint any difference in enzyme antioxidant activity among boars. An additional objective was to determine a potential effect that temperature may have exerted on the level of antioxidant activity.

Experiment 2: Morphological viability post-thaw: In a randomized block design, cryopreserved semen samples from each boar were thawed and co-incubated with their corresponding seminal plasma i.e. autologous or with counterpart SP i.e. homologous. Boar was the random variable to account for between-and-within SP variation among individuals. The endpoint was to measure the effects of SP co-incubations on semen viability and to compare them against the following groups: freeze-thawed only i.e. thawed, freeze-thawedincubated without SP i.e. control, and fresh extended semen.

\section{Semen collection and sample processing}

A total of 48 ejaculates from eight boars (commercial breed Pietrain $\mathrm{x}$ Hampshire) identified as A,B,C,D,E,F,G and $\mathrm{H}$ were obtained by the gloved-hand technique. Additionally, only sperm-rich fractions were collected and filtered through sterile gauze. Each sample with $\geq 70 \%$ gross motility, $\geq 80 \%$ normal morphology and intact acrosome ridges (assessed by phase contrast microscopy) was equally allotted in two aliquots immediately after collection i.e. one for SP collection and the other for semen cryopreservation that was subsequently extended in pre-warmed Vitasem LD $^{\mathrm{ma}}$ (Magapor, Spain; 1:2 v/v). Both samples were transported to the laboratory within $2 \mathrm{hr}$ at $21 \pm 1^{\circ} \mathrm{C}$. To collect SP, raw semen was transferred to $50 \mathrm{ml}$ tubes, cooled at $15^{\circ} \mathrm{C}$ for $120 \mathrm{~min}$ and centrifuged at $800 \times \mathrm{g}$ for $10 \mathrm{~min}$ keeping the same temperature at all times. Thereafter, the supernatant containing the SP was transferred to another tube, centrifuged twice at $800 \times \mathrm{g}$ for $10 \mathrm{~min}$ and stored at $-20^{\circ} \mathrm{C}$ until further use to determine antioxidant enzyme activity or as post-thaw semen co-incubation additive solution.

Semen was cryopreserved in a freezing medium containing $80 \%$ (v/v) Tris-citric acid-glucose solution (111 mM Trizma base, 31.4 $\mathrm{mM}$ monohydrate citric acid, $185 \mathrm{mM}$ glucose) and $20 \%$ egg yolk,

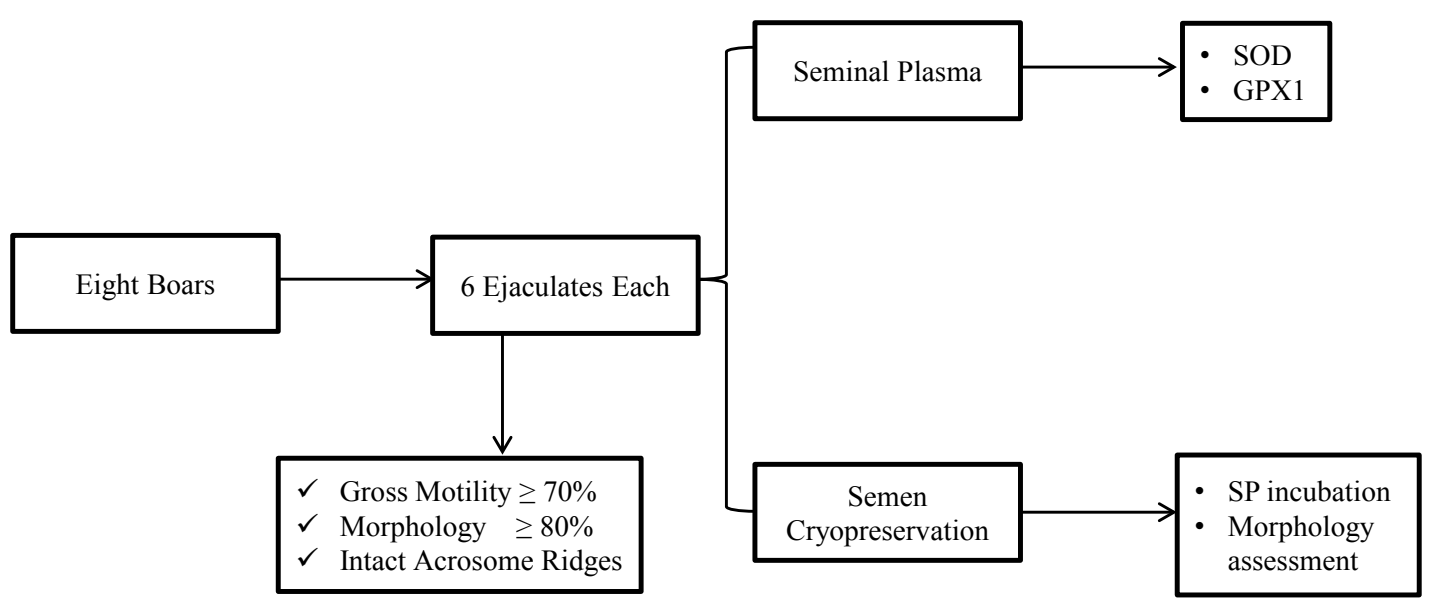

Figure 1: Diagram illustrating the criteria used in sample selection with subsequent experiments on SP enzyme antioxidant activity and sperm morphology variables. 


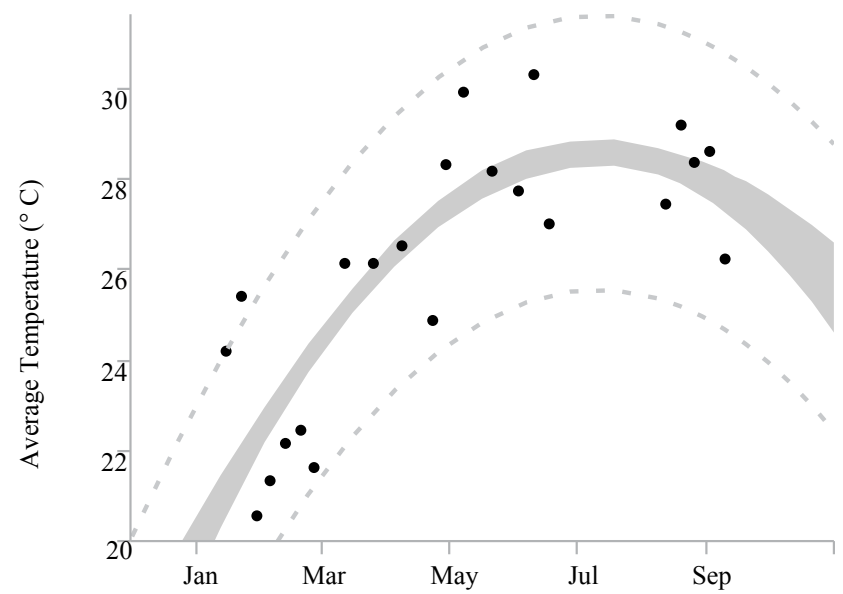

Figure 2: Average temperature at the time of sample collection; a quadratic effect accounted for the typical pattern of temperature observed overtime in Veracruz, Mexico. Dotted lines indicate the individual confidence curve limit and shaded field corresponds to the confidence fit of the model $\left(R^{2}=0.68\right.$; $\mathrm{p}<0.01)$.

supplemented with $100 \mu \mathrm{g} / \mathrm{mL}$ kanamycin sulfate $(\mathrm{pH} 7.2 ; 295-300$ $\mathrm{mOsm} / \mathrm{kg})$. Briefly, upon reception semen samples were centrifuged at $800 \mathrm{~g}$ for $10 \mathrm{~min}$ and the supernatant was discarded. Pelleted semen was immediately diluted in the freezing medium to a concentration of $1200 \times 10^{6}$ sperm per $\mathrm{mL}$ and cooled from $22^{\circ}$ to $4^{\circ} \mathrm{C}$ within 180 min. Freezing medium containing $12 \%$ glycerol was gradually added to the cooled semen within $40 \mathrm{~min}$ (10 min apart) to obtain a final concentration of $6 \%$ glycerol and $600 \times 10^{6}$ sperm per mL. Samples were loaded into $0.5 \mathrm{~mL}$ plastic straws, sealed with PVA and exposed to liquid nitrogen vapor $(4 \mathrm{~cm})$ for $20 \mathrm{~min}$ before plunging them into liquid nitrogen. Stored straws were thawed for $30 \mathrm{~s}$ in a water bath at $37^{\circ} \mathrm{C}$ and treatment samples were supplemented with SP $20 \%(\mathrm{v} / \mathrm{v})$ accordingly.

\section{Morphology assessment}

Vitality was determined using eosin-nigrosin stain (EN; HYSEL ${ }^{\circledR}$, Mexico). Plasma membrane integrity was assessed by the hypoosmotic test (HOST) that consisted on incubating $10 \mu \mathrm{L}$ of semen sample in 1 $\mathrm{mL}$ of a citrate-fructose solution $(150 \mathrm{mOsm} / \mathrm{kg})$ for one hour at $37^{\circ} \mathrm{C}$ and cells with any type of helical twist on the tail were considered as intact. In addition, both tests were carried out in at least three replicates by the same researcher on a 40X magnification with a minimum of 200 cell counts per reading.

\section{SP total protein and enzyme activity assays}

Total protein in the SP was determined by the biuret test where protein bonds were detected in an alkaline solution supplemented with copper ions (Merck ${ }^{\circledR}$, USA) giving violet-colored coordination complexes that were subsequently read by spectrophotometry in $\mathrm{g} /$ $\mathrm{dL}$ [33]. The enzyme activity of SOD was measured by colorimetry using a commercial kit (Ransod ${ }^{\circledR}$ RANDOX $^{\mathrm{TM}}$, USA). Briefly, when provided in solution xanthine oxidase reacts with xanthine and forms superoxide radicals inhibited by the extent of SOD levels. In addition, GPX1 activity was determined by an NADPH-dependent UV kinetic method (Ransel ${ }^{\circledR}$ RANDOX ${ }^{\mathrm{TM}}$, USA) in which cumene hydroperoxideinduced oxidation of glutathione (GSSG) is catalyzed by GPX1. Subsequent reduction to GSH is achieved by providing glutathione reductase and exogenous NADPH yielding NADP+ and the decrease in absorbance was measured. All these procedures were performed as per manufacturers' instructions. Results were expressed in units per milliliter $(\mathrm{U} / \mathrm{mL})$ of SP and subsequently corrected for total SP protein values, thus, enzymatic activity was expressed in units per gram of protein $(\mathrm{U} / \mathrm{g})$.

\section{Statistical analyses}

Analysis of variance (ANOVA) between groups was performed using JMP PRO version 11 (Statistical Discovery from SAS Institute). Percentage (EN or HOST) or numerical (GXP1 or SOD) data not being normally distributed were transformed to arcsine or natural logarithm values, respectively, with appropriate back-transformation for presentation of the results. Intra and inter assay coefficient of variation of GPX1 were $4.73 \%$ and $6.12 \%$, respectively. Likewise, Intra and inter assay coefficient variation of SOD were $5.87 \%$ and $5.91 \%$. We anticipated differences amongst individuals; thus, boar was assumed as the random variable with corresponding restricted maximum likelihood (REML) variance components estimates to account for differences between and within individual. Should the main effect was significant; means were compared using the post hoc test Tukey HSD. A probability of $\mathrm{P}<0.05$ was considered statistically significant and $\mathrm{P}$ $\leq 0.1$ a trend. Since we hypothesized a possible correlation between temperature and enzyme antioxidant activity, we analyzed the readings corresponding to average temperature at collection, SOD and GXP1 with a multivariate model using Spearman's rank correlation coefficient (Spearman's $\rho$ ). Should correlation was strong we plotted the data in a linear regression model to appreciate the effects of temperature on SP antioxidant activity levels.

\section{Results}

\section{Experiment 1: Antioxidant enzyme activity of seminal plasma}

Analysis regarding total protein in seminal plasma (SP) indicated that the average values corresponding to boars $\mathrm{A}, \mathrm{B}$ and $\mathrm{C}$ were numerically higher than the mean $(9.30 \pm 0.39,9.08 \pm 0.69,8.74 \pm$ 0.64 vs. $8.58 \pm 0.30 \mathrm{~g} / \mathrm{dL}$ ). Additionally, REML indicated that there was less variation in SP total protein between than within individual $26.5 \%$ and $73.55 \%$, respectively; however, this effect was not statically significant ( $p>0.05$, Figure 3 ). In terms of SOD, the mean value for its enzyme activity was $9.71 \pm 0.25$ units per gram of SP protein $(\mathrm{U} / \mathrm{g})$. Significant differences were found between boars $\mathrm{G}$ and $\mathrm{H}$ expressing the highest values in SOD activity $(12.01 \pm 0.07 \mathrm{U} / \mathrm{g}, 11.80 \pm 0.12 \mathrm{U} / \mathrm{g})$

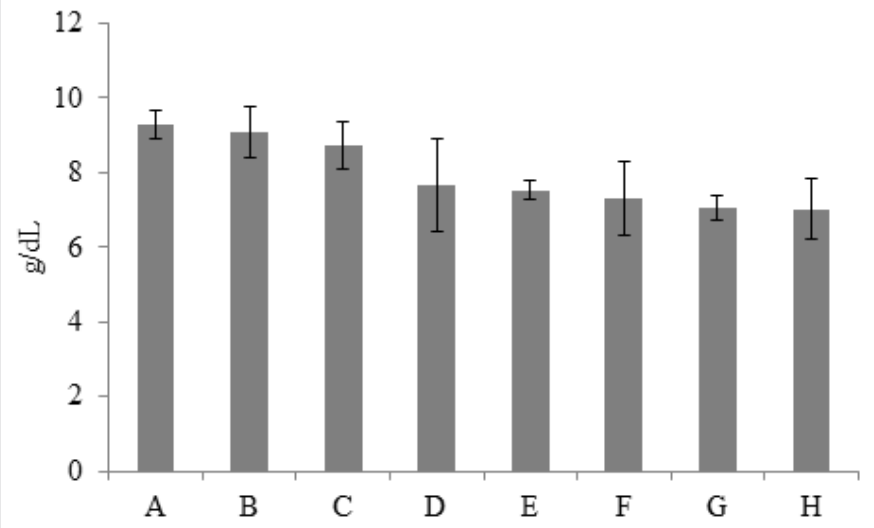

Figure 3: Total protein in seminal plasma expressed in grams per deciliter $(\mathrm{g} / \mathrm{dL})$. Means \pm SEM were no significantly different amongst individuals $(p>0.05)$ 
compared with boars A, B and C $(8.02 \pm 0.12 \mathrm{U} / \mathrm{g}, 7.88 \pm 0.04 \mathrm{U} / \mathrm{g}$, $8.44 \pm 0.02 \mathrm{U} / \mathrm{g}$, respectively). Restricted maximum likelihood variance components estimates indicated that $98 \%$ of the variation resided between individuals ( $\mathrm{p}<0.05$; Figure 4). Furthermore, GPX1 analysis showed that boar E expressed the highest value of antioxidant enzyme activity $(0.05 \pm 0.005 \mathrm{U} / \mathrm{g})$ and it was significantly different compared to boars $\mathrm{A}, \mathrm{B}$ and $\mathrm{C}(0.03 \pm 0.002 \mathrm{U} / \mathrm{g}, 0.03 \pm 0.003 \mathrm{U} / \mathrm{g}$ and $0.03 \pm 0.001$ $\mathrm{U} / \mathrm{g} ; \mathrm{p}<0.05$ ) with a REML between and within individual of $58 \%$ and $42 \%$, respectively (Figure 5 ).

Multivariate correlation analysis did in fact revealed a strong correlation between temperature and SOD and GPX1 enzyme activity levels (main effect $p<0.01$; Spearman's $\rho \geq 0.77$ ). In a subsequent linear regression model we found a positive effect of temperature over SOD and GPX1 $(\mathrm{p}<0.01)$. The first model indicates that one unit increase in average temperature resulted in a $0.44 \pm 0.05 \mathrm{U} / \mathrm{g}$ unit increase of SOD activity. Likewise, a one unit increase in average temperature resulted in $0.003 \pm 0.0005 \mathrm{U} / \mathrm{g}$ unit increase of GPX1 activity (Figure 6).

\section{Experiment 2: Morphological viability post-thaw}

Results indicated that the vitality of the sperm assessed by EN stain was significantly affected by treatment condition. As expected, fresh extended semen showed the highest values compared with thawed and control treatments $(61 \pm 0.36 \%$ vs. $29 \pm 0.28 \%$ and 21 $\pm 0.20 \%$, respectively). However, when semen was supplemented with autologous SP vitality counts were higher than control and not different from homologous SP treatment $(27 \pm 0.59 \%$ and $26 \pm 0.23 \%$; $\mathrm{p}<0.01$; Figure 7). Regarding membrane integrity assessed by HOST, results show a similar trend when compared to EN experiment. Fresh semen showed the highest values as opposed to control with the lowest values $(38 \pm 0.45 \%$ vs. $18 \pm 0.27 \%)$. Further, membrane integrity of sperm treated with autologous and homologous SP were not different from each other and, not different from thawed ones $(28 \pm 0.27 \%, 25 \pm$ $0.13 \%$ and $25 \pm 0.32 \%$, respectively; $p<0.01$; Figure 8 ).

\section{Discussion}

Seminal plasma as an additive to cryopreserved semen has been deemed controversial. In our study, using SP 20\% (v/v), we intended to measure the levels of two important antioxidants i.e. SOD and GPX1 and their effects on sperm morphology variables post-thaw. Another objective was to measure the levels of SP antioxidant activity overtime in a tropical environment. Results on antioxidant enzyme activity are

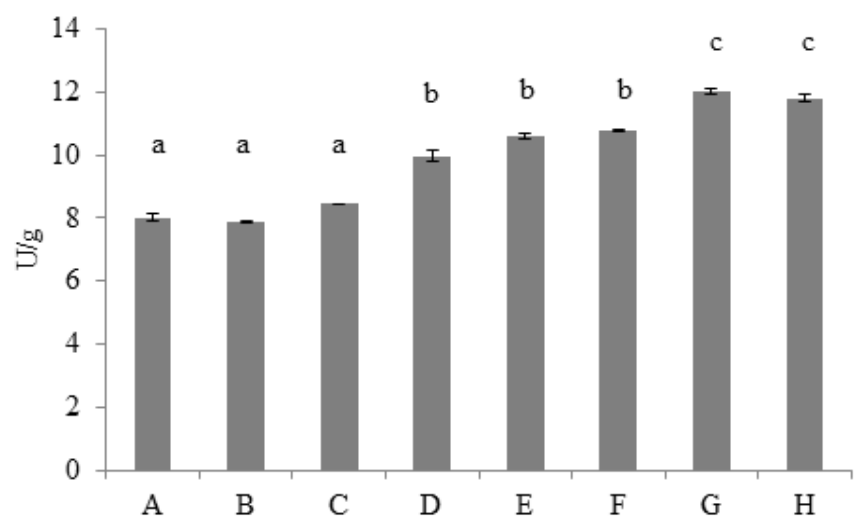

Figure 4: Antioxidant activity of superoxide dismutase (SOD) expressed in units per gram of total SP protein $(U / g)$. Means \pm SEM within a column not sharing the same letter are significantly different $(p<0.05)$.

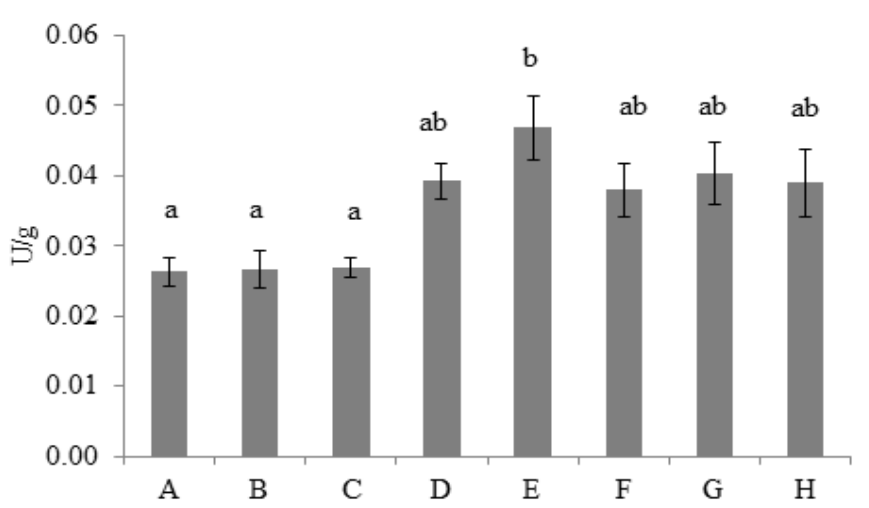

Figure 5: Antioxidant activity of glutathione peroxidase (GPX1) expressed in units per gram of total SP protein $(\mathrm{U} / \mathrm{g})$. Means \pm SEM within a column not sharing the same letter are significantly different $(p<0.05)$.

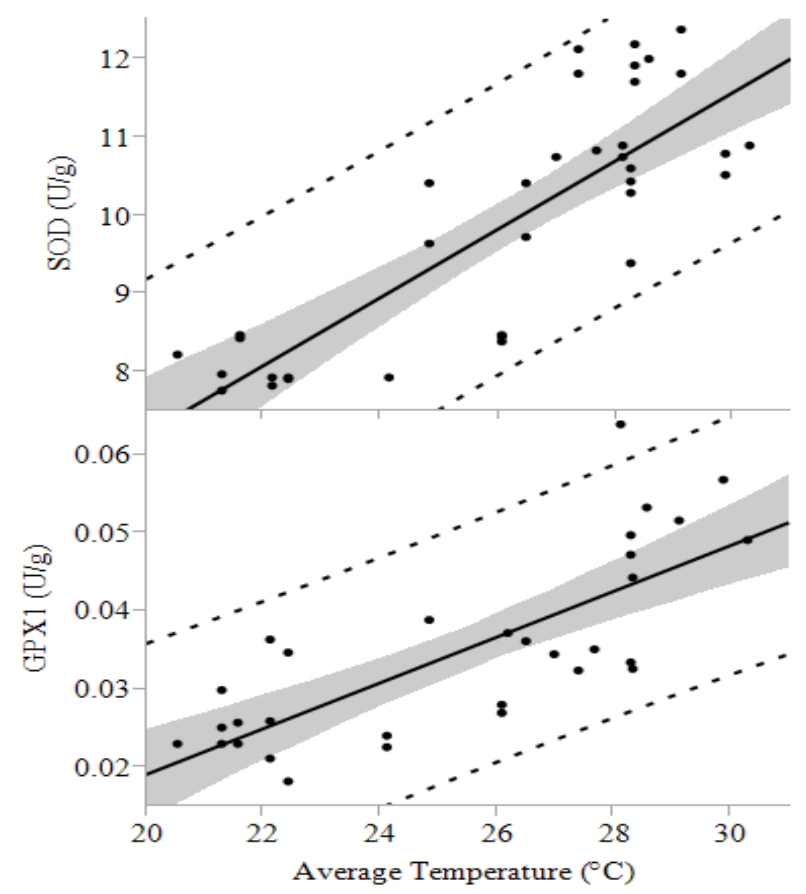

Figure 6: The effect of average temperature on antioxidant enzyme activity of SOD (panel a) and GPX1 (panel b) expressed in units per gram of total $\mathrm{SP}$ protein $(\mathrm{U} / \mathrm{g})$. Data was analyzed by ANOVA in a linear regression model. Dotted lines indicate the individual confidence curve limit and shaded field corresponds to the confidence fit of the model $\left(R^{2}=0.62 ; p<0.01\right)$.

in agreement with those reported elsewhere [34-36] except of those reported by Hernández et al. [23] in which ours seem to differ as per SOD activity. In addition, according to the classification of Hernández et al. [23] boars in this study fall under the category of bad freezers since sperm viability is below $50 \%$ in thawed semen. However, viability in this study was determined by other methods i.e. EN and HOST whereby readings are lower than those using the fluorescent triple stain approach [37]. Moreover, the same authors in Hernández et al. [23] also acknowledged that the enzyme activity does not have an absolute effect on thawed semen viability.

Seminal plasma protein-corrected SOD and GPX1 values were not only different amongst individuals but also enzyme antioxidant activity 


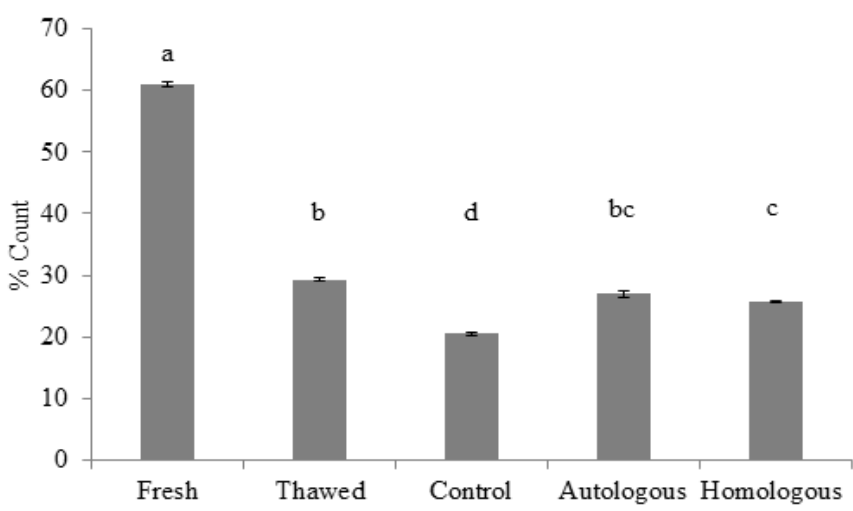

Figure 7: The effect of treatment condition on sperm vitality assessed by nigrosine-eosin stain expressed in percent values. Means \pm SEM within a column not sharing the same letter are significantly different $(p<0.01)$.

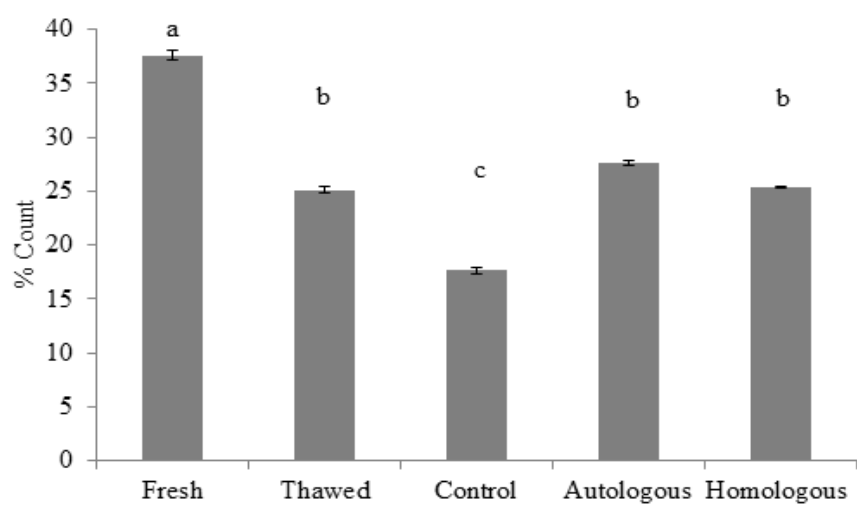

Figure 8: The effect of treatment condition on sperm membrane integrity assessed by HOST expressed in percent values. Means \pm SEM within a column not sharing the same letter are significantly different $(p<0.01)$.

responded to changes in average temperature (Figures 4-6). Individual variation in the quality of SP has been previously reported and findings indicate beneficial effects supplementing SP from good freezer boars to semen with proven history of inferior results [23]. Furthermore, a preliminary study has documented a seasonal-dependent variation in antioxidant activity that results from the vesicular glands sensitivity to changes in temperature independent from antioxidant activity in epididymal fluid since the latter remained constant throughout the year [32]. Indeed, our results show a positive effect of temperature on SOD and GPX1 activity that at the same time seems to have an indirect effect on sperm quality likely to maintain a constant fitness for reproductive success (Figure 6). Addition of SP in a range of 5-50\% (v/v) to the extender minimized cryopreservation-induced membrane damage $[5,9,38]$ or early onset of acrosome responsiveness post-thaw [16,38] due to stabilizing proteins or antioxidant enzymes in SP that provide protection to the sperm cells $[6,13,31]$. Results in our study indicate that addition of SP to frozen-thawed semen ameliorated the negative effects of cryopreservation compared with control sperm as reflected in higher $\mathrm{EN}$ as well as HOST percent values (Figures 7 and 8). A work from Caballero et al. [39] indicated that the addition of $10 \%(\mathrm{v} / \mathrm{v})$ autologous SP consistently improved membrane integrity, mitochondrial function and motility compared to those incubated without PS. However, positive effects from homologous SP exposure depended on boar origin since viability ranged from beneficial to detrimental likely due to an altered concentration of certain components such as stabilizing proteins. In the present study, we found that treatment with either autologous or homologous SP resulted in higher viability rates as compared with control and, no detrimental effects when using the homologous kind. Further, our results are in agreement with those of Kaeoket et al. [14] where addition of autologous or heterologous SP $50 \%(\mathrm{v} / \mathrm{v})$ to extender yielded better viability as well as motility rates than in controls.

It is not new that seminal plasma components are highly variable among individuals $[23,40]$. Indeed, our results show a boar-dependent variation in antioxidant activity. Nonetheless, this variation among individual was controlled for in our statistical models thereby allowing us to detect potential differences when using either autologous or homologous SP supplementation; thus, given the similarity of those results we suspect other factors within SP are affecting the overall quality of sperm. Binder of sperm proteins (BSP; previously known as bovine seminal plasma proteins) have an active role in the biology of the sperm membrane after ejaculation. In bovine species, there are 3 BSPs reported in the binding of the sperm to the tubal epithelium i.e. BSP1, BSP3 and BSP5 and the release thereof during ovulation to acquire acrosome responsiveness prior fertilization of the egg [13]. In addition, BSPs (BSP1 in particular) play a role in sperm membrane configuration including removal of cholesterol and phospholipids during capacitation [13]. Therefore, it is plausible to hypothesize that BSPs are also removed during sperm cryopreservation and adding SP improved viability by restoring some of the lost BSPs.

Antioxidant activity was strongly correlated to temperature but weakly correlated to EN and HOST outcomes (data not shown). These results are in agreement with those of Lasota et al. [41] and Villa et al. [42] reporting a poor correlation between GXP1and SOD on sperm viability. This finding is of particular importance since SOD and GPX1 were correlated to each other based on Spearman's $\rho=0.6(p<0.05)$; hence, other factors in SP may also have contributed to improve sperm viability post-thaw. As mentioned above, GXP1 is one of the most important peroxidases within the Glutathione redox system. The second, but not least, important redox system in eukaryote cells is the Thioredoxin system composed by NADPH, Thioredoxin reductases (TRXR1 in particular) and thioredoxin (TRX; [43]). GSH and TRX systems interact with each other albeit they are not mutually exclusive; they are the most important antioxidant systems against oxidative stress. Since both GPXs and TRXRs are selenoenzymes more research is needed to unveil a possible interaction in the seminal plasma, because the former, as the primary redox buffer, is competing with the TRX system for reducing equivalents. Furthermore, it may be worth to investigate other thiol oxidoreductases that participate conjointly within the GSH/TRX systems not only in the control of oxidative stress but also in the prevention of protein misfolding in which the ubiquitinproteasome system is also involved [44,45].

\section{Conclusion}

Herein, adding seminal plasma to frozen-thawed semen has resulted in improved morphological viability assessed by EN and HOST. Additionally, SOD and GPX1 enzymatic activity was influenced by average temperature. Since SP antioxidant enzyme activity did not have a strong effect on viability per se, we believe an unknown factor(s) played a significant role towards improved viability post-thaw. It seems possible that a stabilizing protein i.e. BSP or an additional redox buffer system i.e. TRX may better explain the variation in morphological viability of cryopreserved semen in swine species. Further research is needed to answer those questions. 
Citation: Hernández DAK, Barrientos-Morales M, Cervantes AP, Hernández BA, Domínguez MB, et al. (2015) Antioxidant Effects of Seminal Plasma on Cellular Morphological Viability of Swine Semen Post-Cryopreservation. J Veterinar Sci Technol 6: 225. doi:10.4172/2157-7579.1000225

\section{Acknowledgements}

The authors appreciate all the uncompensated support provided by the participating farms and Swine Producer Association of Emiliano Zapata, Veracruz Mexico. We gratefully acknowledge CONACyT-Mexico for the funding provided (Grant 169861) and CONACyT scholarships to partially support DAK Hernández MS program.

\section{References}

1. Gadea J, García-Vazquez F, Matás C, Gardón JC, Cánovas S, et al. (2005) Cooling and freezing of boar spermatozoa: supplementation of the freezing media with reduced glutathione preserves sperm function. J Androl 26: 396404.

2. Sellés E, Gadea J, Romar R, Matás C, Ruiz S (2003) Analysis of in vitro fertilizing capacity to evaluate the freezing procedures of boar semen and to predict the subsequent fertility. Reprod Domest Anim 38: 66-72.

3. Roca J, Rodríguez MJ, Gil MA, Carvajal G, Garcia EM, et al. (2005) Survival and in vitro fertility of boar spermatozoa frozen in the presence of superoxide dismutase and/or catalase. J Androl 26: 15-24

4. Barbas JP, Mascarenhas RD (2009) Cryopreservation of domestic animal sperm cells. Cell Tissue Bank 10: 49-62.

5. Vadnais ML, Kirkwood RN, Specher DJ, Chou K (2005) Effects of extender incubation temperature, and added seminal plasma on capacitation of cryopreserved, thawed boar sperm as determined by chlortetracycline staining. Anim Reprod Sci 90: 347-354.

6. Vadnais ML, Roberts KP (2007) Effects of seminal plasma on cooling-induced capacitative changes in boar sperm. J Androl 28: 416-422.

7. Green CE, Watson PF (2001) Comparison of the capacitation-like state of cooled boar spermatozoa with true capacitation. Reproduction 122: 889-898.

8. Vadnais ML, Althouse GC (2011) Characterization of capacitation, cryoinjury, and the role of seminal plasma in porcine sperm. Theriogenology 76: 15081516 .

9. Brezezi A, Ska-Slebodzi A, Ska E, Slebodzi A, Ski AB, et al. (1995) Antioxidan effect of vitamin $E$ and glutathione on lipid peroxidation in boar semen plasma. Biol Trace Elem Res 47: 69-74.

10. Suzuki K, Asano A, Eriksson B, Niwa K, Nagai T, et al. (2002) Capacitation status and in vitro fertility of boar spermatozoa: effects of seminal plasma, cumulus-oocyte-complexes-conditioned medium and hyaluronan. Int $\mathrm{J}$ Androl 25: 84-93.

11. Bailey JL, Lessard C, Jacques J, Brèque C, Dobrinski I, et al. (2008) Cryopreservation of boar semen and its future importance to the industry. Theriogenology 70: 1251-1259.

12. Strzeek J, Wysocki P, Kordan W, Kukliska M, Mogielnicka M, et al. (2005) Proteomics of boar seminal plasma - current studies and possibility of thei application in biotechnology of animal reproduction. Reprod Biol 5: 279-290.

13. Hung PH, Suarez SS (2012) Alterations to the bull sperm surface proteins that bind sperm to oviductal epithelium. Biol Reprod 87: 88

14. Kaeoket $K$, Chanapiwat $P$, Tummaruk $P$, Techakumphu $M$, Kunavongkrit $A$ (2011) A preliminary study on using autologous and heterologous boar sperm supernatant from freezing processes as post-thawing solution: its effect on sperm motility. Trop Anim Health Prod 43: 1049-1055.

15. Radomil L, Pettitt MJ, Merkies KM, Hickey KD, Buhr MM (2011) Stress and dietary factors modify boar sperm for processing. Reprod Domest Anim 46 Suppl 2: 39-44

16. Bailey JL, Bilodeau JF, Cormier N (2000) Semen cryopreservation in domestic animals: a damaging and capacitating phenomenon. J Androl 21: 1-7.

17. Cerolini S, Maldjian A, Pizzi F, Gliozzi TM (2001) Changes in sperm quality and lipid composition during cryopreservation of boar semen. Reproduction 121 395-401.

18. Saleh RA, Agarwal A (2002) Oxidative stress and male infertility: from research bench to clinical practice. J Androl 23: 737-752.

19. de Graaf SP, Leahy T, Marti J, Evans G, Maxwell WM (2008) Application of seminal plasma in sex-sorting and sperm cryopreservation. Theriogenology 70 : 1360-1363.
20. Juarez JD, Parrilla I, Vazquez JM, Martinez EA, Roca J (2011) Boar semen can tolerate rapid cooling rates prior to freezing. Reprod Fertil Dev 23: 681-690.

21. Eriksson BM, Vazquez JM, Martinez EA, Roca J, Lucas X, et al. (2001) Effects of holding time during cooling and of type of package on plasma membrane integrity, motility and in vitro oocyte penetration ability of frozen-thawed boar spermatozoa. Theriogenology 55: 1593-1605.

22. Yeste M, Estrada E, Rivera Del Álamo MM, Bonet S, Rigau T, et al. (2014) The increase in phosphorylation levels of serine residues of protein HSP70 during holding time at $17^{\circ} \mathrm{C}$ is concomitant with a higher cryotolerance of boar spermatozoa. PLoS One 9: e90887.

23. Hernández M, Roca J, Calvete JJ, Sanz L, Muiño-Blanco T, et al. (2007) Cryosurvival and in vitro fertilizing capacity postthaw is improved when boar spermatozoa are frozen in the presence of seminal plasma from good freezer boars. J Androl 28: 689-697.

24. Saravia F, Wallgren M, Nagy S, Johannisson A, Rodríguez-Martínez H (2005) Deep freezing of concentrated boar semen for intra-uterine insemination: effects on sperm viability. Theriogenology 63: 1320-1333.

25. Tomás C, Gómez-Fernández J, Gómez-Izquierdo E, de Mercado E (2014) Effect of the holding time at $15{ }^{\circ} \mathrm{C}$ prior to cryopreservation, the thawing rate and the post-thaw incubation temperature on the boar sperm quality after cryopreservation. Anim Reprod Sci 144: 115-121.

26. Guthrie HD, Welch GR (2005) Impact of storage prior to cryopreservation on plasma membrane function and fertility of boar sperm. Theriogenology 63: 396410.

27. Okazaki T, Abe S, Yoshida S, Shimada M (2009) Seminal plasma damages sperm during cryopreservation, but its presence during thawing improves semen quality and conception rates in boars with poor post-thaw semen quality. Theriogenology 71: 491-498.

28. Absalón-Medina VA, Butler WR, Gilbert RO (2014) Preimplantation embryo metabolism and culture systems: experience from domestic animals and clinical implications. J Assist Reprod Genet 31: 393-409.

29. Gadea J, Sellés E, Marco MA, Coy P, Matás C, et al. (2004) Decrease in glutathione content in boar sperm after cryopreservation. Effect of the addition of reduced glutathione to the freezing and thawing extenders. Theriogenology 62: 690-701.

30. Zhu J, Xu X, Cosgrove JR, Foxeroft GR (2000) Effects of semen plasma from different fractions of individual ejaculates on IVF in pigs. Theriogenology 54 $1443-1452$.

31. Pech-Sansores AGC, Centurión-Castro FG, Rodríguez-Buenfil JC (2011) Effect of the addition of seminal plasma, vitamin $E$ and incubation time on postthawed sperm viability in boar semen. Tropical and subtropical agroecosystems 14: $965-971$

32. Dziekoska A, Fraser L, Koziorowska-Gilun M, Strzezek J, Koziorowski M, et al. (2014) Seasonal-dependent variations in metabolic status of spermatozoa and antioxidant enzyme activity in the reproductive tract fluids of wild boar/domestic pig hybrids. Pol J Vet Sci 17: 307-313

33. Smith PK, Krohn RI, Hermanson GT, Mallia AK, Gartner FH, et al. (1985) Measurement of protein using bicinchoninic acid. Anal Biochem 150: 76-85.

34. Kowalowka M, Wysocki P, Fraser L, Strzezek J (2008) Extracellular superoxide dismutase of boar seminal plasma. Reprod Domest Anim 43: 490-496.

35. Koziorowska-Gilun M, Koziorowski M, Fraser L, Strzeek J (2011) Antioxidant defence system of boar cauda epididymidal spermatozoa and reproductive tract fluids. Reprod Domest Anim 46: 527-533.

36. Kolodziej A, Jacyno E (2004) Effect of Dietary Selenium and Vitamin E Supplementation on Reproductive Performance of Young Boars. Electronic Journal of Polish Agricultural Universities 7: 4

37. Wu TW, Cheng FP, Chen IH, Yang CH, Tsai MY, et al. (2013) The combinatoria effect of different Equex STM paste concentrations, cryoprotectants and the straw-freezing methods on the post-thaw boar semen quality. Reprod Domest Anim 48: 53-58

38. Carvajal G, Cuello C, Ruiz M, Vázquez JM, Martínez EA, et al. (2004) Effects of centrifugation before freezing on boar sperm cryosurvival. J Androl 25: 389396

39. Caballero I, Vazquez JM, Centurión F, Rodríguez-Martinez H, Parrilla I, et al (2004) Comparative effects of autologous and homologous seminal plasma on 
Citation: Hernández DAK, Barrientos-Morales M, Cervantes AP, Hernández BA, Domínguez MB, et al. (2015) Antioxidant Effects of Seminal Plasma on Cellular Morphological Viability of Swine Semen Post-Cryopreservation. J Veterinar Sci Technol 6: 225. doi:10.4172/2157-7579.1000225

the viability of largely extended boar spermatozoa. Reprod Domest Anim 39: 370-375

40. Roca J, Rodŕiguez-Martínez H, Vázquez JM, Bolarín A, Hernández M, (2006) Strategies to improve the fertility of frozen-thawed boar semen for artificial insemination. Soc Reprod Fertil Suppl 62: 261-275.

41. Lasota B, Baszczyk B, Seremak B, Udaa J (2004) Selenium status and GSHPx activity in semen and blood of boars at different ages used for artificial insemination. Reprod Domest Anim 39: 309-314.

42. Villa NA, Castaño D, Duque PC, Ceballos A (2012) Glutathione Peroxidase and Superoxide Dismutase activities in blood and seminal plasma in colombian stallions. Revista Colombiana de Ciencias Pecuarias 25: 64-70.
43. Lu J, Holmgren A (2014) The thioredoxin antioxidant system. Free Radic Bio Med 66: 75-87.

44. Barnea ER, Lubman DM, Liu YH, Absalon-Medina V, Hayrabedyan S, et al (2014) Insight into Prelmplantation Factor (PIF*) mechanism for embryo protection and development: target oxidative stress and protein misfolding (PDI and HSP) through essential RIPK binding site. PLoS One 9: e100263.

45. Yi YJ, Sutovsky M, Song WH, Sutovsky P (2014) Protein deubiquitination during oocyte maturation influences sperm function during fertilisation, antipolyspermy defense and embryo development. Reprod Fertil Dev. 